\title{
Linear Analysis of an Integro-Differential Delay Equation Model
}

\author{
Anael Verdugo $\mathbb{D D}^{1,2}$ \\ ${ }^{1}$ Department of Mathematics, California State University, Fullerton, CA, USA \\ ${ }^{2}$ Center for Computational and Applied Mathematics, California State University, Fullerton, CA, USA
}

Correspondence should be addressed to Anael Verdugo; averdugo@fullerton.edu

Received 2 December 2017; Accepted 14 March 2018; Published 2 May 2018

Academic Editor: Elena Braverman

Copyright (C) 2018 Anael Verdugo. This is an open access article distributed under the Creative Commons Attribution License, which permits unrestricted use, distribution, and reproduction in any medium, provided the original work is properly cited.

\begin{abstract}
This paper presents a computational study of the stability of the steady state solutions of a biological model with negative feedback and time delay. The motivation behind the construction of our system comes from biological gene networks and the model takes the form of an integro-delay differential equation (IDDE) coupled to a partial differential equation. Linear analysis shows the existence of a critical delay where the stable steady state becomes unstable. Closed form expressions for the critical delay and associated frequency are found and confirmed by approximating the IDDE model with a system of $N$ delay differential equations (DDEs) coupled to $N$ ordinary differential equations. An example is then given that shows how the critical delay for the DDE system approaches the results for the IDDE model as $N$ becomes large.
\end{abstract}

\section{Introduction}

New genetic experiments [1-3] and mathematical approaches [4-6] have been developed to help us better understand how genes interact within a cell. Theoretically, the structure of these interactions or networks are represented by the various chemical reactions happening at a certain time. If the reactions under consideration only involve a few genes, then their dynamic behavior could be understood intuitively and, most likely, confirmed with a biochemical experiment $[2,3]$. However, if the system is formed of dozens of reactions, then developing an intuitive understanding of the system's dynamics would be difficult. Nevertheless, current research in the computational sciences $[7,8]$ shows that the study of these large gene networks is an important step which will help us unravel some of the mysteries in the field of cell biology $[5,6]$.

An important and popular modeling technique in the applied sciences is based on differential equations in all its various forms: linear [9], nonlinear [6, 10], partial [11], stochastic $[12,13]$, and delayed $[3,6,14]$. In this study we focus our attention to a differential equation model with constant delay, where the delay arises naturally as the time lag associated with various intracellular processes, like movement within the cell, synthesis of proteins, and transcription of DNA, among many others. The model that motivated this work was studied previously by [4-6] and is given by the following set of delay differential equations (DDEs):

$$
\begin{aligned}
& \frac{d m}{d t}=-\mu_{m} m(t)+H(p(t-T)), \\
& \frac{d p}{d t}=m(t)-\mu_{p} p(t),
\end{aligned}
$$

where the time dependent variables are the mRNA concentration, $m(t)$, and its associated protein concentration, $p(t)$, and the constants $\mu_{m}$ and $\mu_{p}$ are the decay rates of the mRNA and protein molecules, respectively. The function $H(p(t-T))$ is generally a Hill equation representing the rate of delayed production of mRNA, where the delay, $T$, is assumed to be a positive constant. The associated biochemical representation of the system is given in Figure 1(a) and the biological context is the following: a gene is copied onto mRNA in the nucleus, which is then translated into a protein in the cytoplasm of the cell. The protein then returns to the nucleus and acts as a negative feedback regulator by repressing production of mRNA (see [4-6] for more biological background).

In this paper, we analyze the steady state stability of a model motivated by (1) and previously studied by the author in [15]. The model is given by an integro-delay differential equation (IDDE) coupled to a partial differential equation 


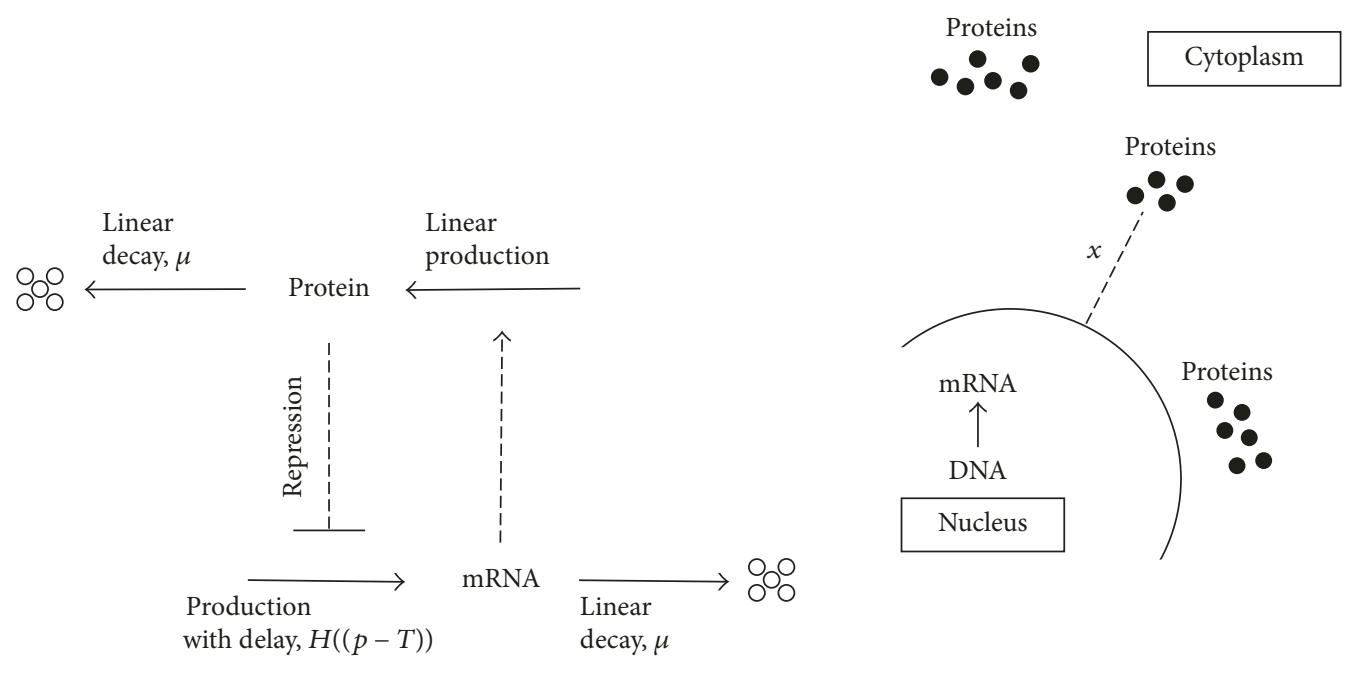

(a)

(b)

Figure 1: (a) Biological circuit diagram of (1). Protein production, protein decay, and mRNA decay are assumed to be linear processes. Production of mRNA is considered as a process affected by a delayed response of protein repression. Here the arrow $(\uparrow)$ represents activation and the perpendicular symbol $(\perp)$ represents repression. Solid and dashed lines represent direct chemical reactions and indirect regulatory signals, respectively. Five small circles represent degradation byproducts. (b) Spatial distribution of protein production in the cytoplasm. Protein synthesis happens at various locations from the nucleus. The distance from the nucleus is represented here by the variable $x$, where $0 \leq x \leq 1$.

(PDE) and is characterized by an exponential "weighting" function that regulates the net repression effect on mRNA based on protein synthesis location. The model is given by

$$
\begin{aligned}
\frac{d m}{d t} & =-\mu m+\int_{0}^{1} e^{-|x-\bar{x}|} H(p(\bar{x}, t-T)) d \bar{x}, \\
\frac{d p}{d t} & =m-\mu p,
\end{aligned}
$$

where $m=m(x, t), p=p(x, t)$, and $e^{-|x-\bar{x}|}$ is a weighting function that accounts for a "stronger" mRNA repression for proteins being synthesized closer to the nucleus than more distant ones. The latter is due to the spatial distribution of protein production within the cytoplasm, which occurs after mRNA exits the nucleus and diffuses into the cytoplasm where it is caught, read, and translated into a protein. The exact location from the nucleus where this process occurs is arbitrary and here we quantify it with a distance variable $0 \leq x \leq 1$ as explained in Figure 1(b). The latter yields the integral term in (2) which represents the total sum of the repression effect that newly synthesized proteins have on mRNA.

The current work extends our previous study [15] in two different ways. First, the biological setup explained above sets our model (2)-(3) on firmer modeling grounds from our previous study [15]. Here we assume the variable $x$ is "distance" from the nucleus, as opposed to $x$ being a variable that represents gene sites in the DNA as argued in [15]. This is a crucial difference that yields a better understanding of our computational results. Second, the results from the analysis of the steady state and its associated stability are now confirmed via MATLAB's dde23.m, which provides more accurate approximations and numerical simulations for the associated $2 \mathrm{~N}$-dimensional system. The latter was not accomplished in [15] and thus presented here for the first time.

The rest of the paper is organized as follows. In Section 2, we present the associated linear stability analysis of (2)-(3) and characterize the steady state solutions. Linear analysis reveals the existence of a critical delay where the stable steady state becomes unstable and thus closed form expressions for the critical delay, $T_{\mathrm{cr}}$, and associated frequency $\omega$ are found. In Section 3, we construct a system of $N$ DDEs coupled to $N$ ordinary differential equations (ODEs) and use these to confirm the results obtained in Section 2. A numerical example is then given in Section 4, which shows how the critical delay for the DDE system approaches the results for the IDDE model as $N$ becomes large. In Section 5, we discuss our findings and conclusions.

\section{Linear Stability Analysis}

In this section, we consider the steady state behavior of (2) and (3). Setting $d m / d t=d p / d t=0$, we see from (3) that at steady state $m^{*}=\mu p^{*}$, where $\left(m^{*}(x), p^{*}(x)\right)$ represents the steady state solution. Substituting the latter into (2) gives

$$
\mu^{2} p^{*}(x)=\int_{0}^{1} e^{-|x-\bar{x}|} H\left(p^{*}(\bar{x})\right) d \bar{x} .
$$

Splitting the integration limits

$$
\begin{aligned}
\mu^{2} p^{*}(x)= & e^{-x} \int_{0}^{x} e^{\bar{x}} H\left(p^{*}(\bar{x})\right) d \bar{x} \\
& +e^{x} \int_{x}^{1} e^{-\bar{x}} H\left(p^{*}(\bar{x})\right) d \bar{x}
\end{aligned}
$$


and differentiating twice, we obtain an equivalent secondorder two-point boundary value problem (BVP) for the equilibrium solution $p^{*}=p^{*}(x)$

$$
\frac{d^{2} p^{*}}{d x^{2}}=p^{*}-\frac{2}{\mu^{2}} H\left(p^{*}\right),
$$

where the boundary conditions (BCs) are given by

$$
\begin{aligned}
& p^{*}(0)=\frac{1}{\mu^{2}} \int_{0}^{1} e^{-\bar{x}} H\left(p^{*}(\bar{x})\right) d \bar{x} \\
& p^{*}(1)=\frac{1}{e \mu^{2}} \int_{0}^{1} e^{\bar{x}} H\left(p^{*}(\bar{x})\right) d \bar{x} .
\end{aligned}
$$

The BVP (6)-(7) has a unique solution as long as the right hand side (RHS) of (6) has bounded, positive, and continuous partial derivatives with respect to $p^{*}$. For the rest of this work, we let

$$
H(p(x, t))=1-p(x, t)
$$

which allows mathematical tractability for the stability analysis presented below. Notice that since (8) satisfies all three aforementioned BVP conditions, then we are guaranteed the existence of a unique solution, which can be approximated using a numerical technique for BVPs, such as finite differences or a shooting method. See Section 4 for an example.

To study the stability of the steady state solution $\left(m^{*}(x), p^{*}(x)\right)$, we set $p(x, t)=p^{*}(x)+\eta(x, t)$ and $m(x, t)=$ $m^{*}(x)+\xi(x, t)$, substitute these into (2)-(3), and linearize the resulting equations in $\eta(x, t)$ and $\xi(x, t)$ to obtain

$$
\begin{aligned}
& \frac{d \xi}{d t}=-\mu \xi-\int_{0}^{1} e^{-|x-\bar{x}|} \eta_{d}(\bar{x}) d \bar{x}, \\
& \frac{d \eta}{d t}=\xi-\mu \eta .
\end{aligned}
$$

Setting $\xi(x, t)=\phi(x) e^{\lambda t}$ and $\eta(x, t)=\psi(x) e^{\lambda t}$ gives

$$
\begin{aligned}
-e^{\lambda T}(\lambda+\mu) \phi(x) & =\int_{0}^{1} e^{-|x-\bar{x}|} \psi(\bar{x}) d \bar{x}, \\
(\lambda+\mu) \psi(x) & =\phi(x),
\end{aligned}
$$

which yields

$$
r \psi(x)=\int_{0}^{1} e^{-|x-\bar{x}|} \psi(\bar{x}) d \bar{x},
$$

where the RHS has a symmetric integral kernel, $\psi(x)$ is an eigenfunction, and $r$ is the associated eigenvalue given by

$$
r=-e^{\lambda T}(\lambda+\mu)^{2}
$$

Since (12) is a self-adjoint operator of the form

$$
L(\cdot)=\int_{0}^{1} K(x, \bar{x})(\cdot) d \bar{x},
$$

then the eigenvalue problem (12) has real eigenvalues $r \in \mathbb{R}$. To compute $r$, we transform the integral equation (12) to the following equivalent second-order BVP:

$$
\frac{d^{2} \psi}{d x^{2}}+\rho \psi=0
$$

with solutions

$$
\psi(x)=c_{1} \sin (\rho x)+c_{2} \cos (\rho x),
$$

where $c_{1}$ and $c_{2}$ are constants and $\rho=\sqrt{2 / r-1}$. The endpoint $\mathrm{BCs}$ are obtained from (12) as follows:

$$
\begin{aligned}
& \psi(0)=\frac{\rho^{2}+1}{2} \int_{0}^{1} e^{-\bar{x}} \psi(\bar{x}) d \bar{x}, \\
& \psi(1)=\frac{\rho^{2}+1}{2 e} \int_{0}^{1} e^{\bar{x}} \psi(\bar{x}) d \bar{x} .
\end{aligned}
$$

Substituting the solution (16) into the BCs (17) gives the system of equations

$$
\begin{aligned}
& {\left[\begin{array}{cc}
\rho \sin \rho-\cos \rho-e & -\sin \rho-\rho \cos \rho+e \rho \\
e \rho \sin \rho-e \cos \rho-1 & -e \sin \rho-e \rho \cos \rho+\rho
\end{array}\right]\left[\begin{array}{l}
c_{1} \\
c_{2}
\end{array}\right]} \\
& \quad=0
\end{aligned}
$$

which yields the condition on $\rho$ for nontrivial solutions

$$
\left(\rho^{2}-1\right) \sin \rho-2 \rho \cos \rho=0 .
$$

Using a numerical root finding technique on (19), we obtain $\rho=1.30654,3.67319,6.58462, \ldots$ which gives the corresponding values for $r=2 /\left(1+\rho^{2}\right)=0.73881,0.13800$, $0.04509, \ldots$. Thus to determine $\lambda$ from $r$ we have two cases:

(i) For $T=0$, (13) gives $\lambda=-\mu \pm \sqrt{-r}$ and since $r=$ $2 /\left(1+\rho^{2}\right)>0$ then $\operatorname{Re}(\lambda)=-\mu<0$ for $\mu>0$. The latter shows that the equilibrium solution is stable when there is no delay.

(ii) For $T=T_{\mathrm{cr}}$ and $\lambda=i \omega$, (13) becomes $r=-e^{i \omega T_{\mathrm{cr}}}(i \omega+$ $\mu)^{2}$ which gives the two real equations

$$
\begin{aligned}
& r=2 \mu \omega \sin \left(\omega T_{\mathrm{cr}}\right)+\left(\omega^{2}-\mu^{2}\right) \cos \left(\omega T_{\mathrm{cr}}\right), \\
& 0=\left(\omega^{2}-\mu^{2}\right) \sin \left(\omega T_{\mathrm{cr}}\right)-2 \mu \omega \cos \left(\omega T_{\mathrm{cr}}\right) .
\end{aligned}
$$

Solving (20) for $\sin \left(\omega T_{\mathrm{cr}}\right)$ and $\cos \left(\omega T_{\mathrm{cr}}\right)$, and using the identity $\sin ^{2}\left(\omega T_{\mathrm{cr}}\right)+\cos ^{2}\left(\omega T_{\mathrm{cr}}\right)=1$, we obtain

$$
\omega=\sqrt{r-\mu^{2}}
$$

Dividing the expressions for $\sin \left(\omega T_{\mathrm{cr}}\right)$ and $\cos \left(\omega T_{\mathrm{cr}}\right)$ and solving for $T_{\mathrm{cr}}$, we obtain

$$
T_{\mathrm{cr}}=\frac{1}{\sqrt{r-\mu^{2}}} \arctan \left(\frac{2 \mu \sqrt{r-\mu^{2}}}{r-2 \mu^{2}}\right),
$$


which gives the value of the delay where the equilibrium solution loses stability. The smallest value for $T_{\text {cr }}$ is obtained by setting $r=0.73881$ to obtain an expression in terms of the decay rate $\mu$. In Section 4 , we present a numerical example to confirm these results.

\section{Approximating the IDDE-PDE Equations with a DDE-ODE System}

To check our previous results, we "discretize" the variables $\xi(x, t)$ and $\eta(x, t)$ in (9) and (10) with a set of $2 N$ variables $\xi_{i}(t)$ and $\eta_{i}(t)$ for $i=1,2, \ldots, N$. This replaces the original model (9) and (10) with a $2 N$-dimensional system of $N$ DDEs coupled to $N$ ODEs and replaces the integral in (9) with a sum of $N$ terms as follows:

$$
\begin{aligned}
& \dot{\xi}_{i}=-\mu \xi_{i}-\frac{1}{N} \sum_{j=1}^{N} e^{-|i-j| / N} \eta_{j}(t-T), \\
& \dot{\eta}_{i}=\xi_{i}-\mu \eta_{i},
\end{aligned}
$$

where $i=1,2, \ldots, N$. By assuming solutions of the form $\xi_{i}=$ $\phi_{i} e^{\lambda t}$ and $\eta_{i}=\psi_{i} e^{\lambda t}$ and substituting them into (23), we obtain

$$
\begin{aligned}
-e^{\lambda T}(\lambda+\mu) \phi_{i} & =\frac{1}{N} \sum_{j=1}^{N} e^{-|i-j| / N} \psi_{j}, \\
(\lambda+\mu) \psi_{i} & =\phi_{i},
\end{aligned}
$$

which yields the following eigenvalue problem:

$$
c \psi_{i}=\sum_{j=1}^{N} e^{-|i-j| / N} \psi_{j}
$$

where $c=N r$ and $r=-e^{\lambda T}(\lambda+\mu)^{2}$. For nontrivial solutions, system (25) of $N$ equations must satisfy $\operatorname{det}(K-c I)=0$, where $K$ is the $N \times N$ matrix $K=\left[e^{-|i-j| / N}\right]$ and $c$ is its associated eigenvalue. Since $K$ is a symmetric matrix, then all of its eigenvalues are real. Furthermore, $K$ is positive definite because $\operatorname{det}\left(M_{i i}\right)>0$ for $i=1,2, \ldots, N$, where $M_{i i}$ is the $i$ th minor of $K$ along the main diagonal. Hence $K$ is a symmetric positive definite matrix, which shows that $c$ is a positive real number. The steady state stability results are thus summarized as follows:

(i) For $T=0$, we have that $\lambda=-\mu \pm \sqrt{-c / N}$, where $\mu, N, c>0$. This shows that $\operatorname{Re}(\lambda)<0$ and so the equilibrium solution with no delay is stable.

(ii) For $T=T_{\mathrm{cr}}$, we take the smallest value of $c$ for any given $N$ and use (21) and (22) to obtain values for $\omega$ and $T_{\text {cr }}$ where we set $r=c / N$. A numerical example of this case is presented in the following section.

\section{Numerical Example}

In this section, we present a numerical example to compare and confirm our previous results. From (6) and (8), we obtain

$$
\frac{d^{2} p^{*}}{d x^{2}}-\gamma p^{*}=1-\gamma
$$

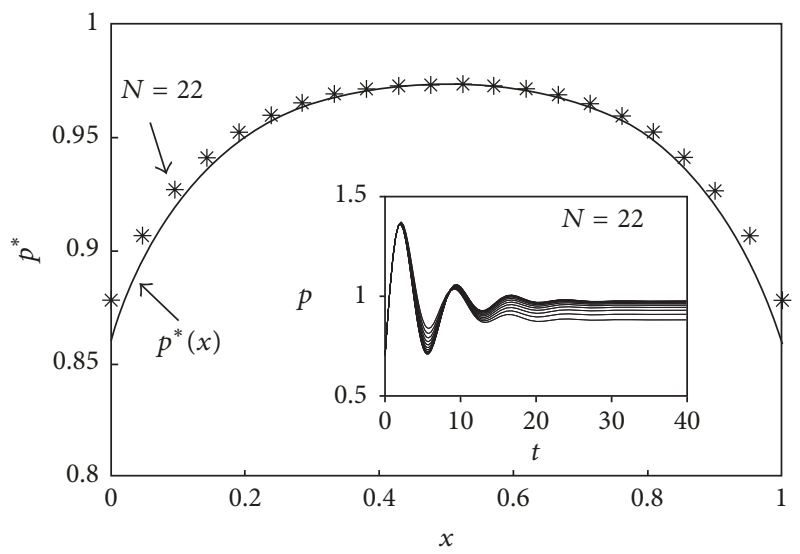

FIGURE 2: Numerical comparison between the steady state solutions for (29) and the 44-dimensional system given by (30). Here we also present a time course simulation for $N=22$, which shows the short transient response to equilibrium. The asterisk marks $(*)$ are the numerical values extracted from $t=40$ in the time course simulation for the 22 variables $p_{i}$ for $i=1,2, \ldots, 22$.

where $\gamma=1+2 / \mu^{2}>0$ gives the following solution:

$$
p^{*}(x)=c_{1} \sinh (\sqrt{\gamma} x)+c_{2} \cosh (\sqrt{\gamma} x)+\frac{2}{\mu^{2} \gamma} \text {. }
$$

Substituting (27) into the BCs (7), we obtain

$$
\begin{aligned}
c_{1}= & \left(1-e^{\sqrt{\gamma}}\right) \\
& \cdot\left(\frac{1-\sqrt{\gamma}-(1+\sqrt{\gamma}) e^{\sqrt{\gamma}}}{\gamma\left[\left(\mu^{2} \sqrt{\gamma}+\mu^{2}+1\right) e^{2 \sqrt{\gamma}}+\mu^{2} \sqrt{\gamma}-\mu^{2}-1\right]}\right), \\
c_{2}= & \left(1+e^{\sqrt{\gamma}}\right) \\
& \cdot\left(\frac{1-\sqrt{\gamma}-(1+\sqrt{\gamma}) e^{\sqrt{\gamma}}}{\gamma\left[\left(\mu^{2} \sqrt{\gamma}+\mu^{2}+1\right) e^{2 \sqrt{\gamma}}+\mu^{2} \sqrt{\gamma}-\mu^{2}-1\right]}\right) .
\end{aligned}
$$

Letting $\mu=0.2$, we obtain

$$
\begin{aligned}
p^{*}(x)= & 0.12 \sinh (7.14 x)-0.12 \cosh (7.14 x) \\
& +0.98
\end{aligned}
$$

which we have plotted in Figure 2 (solid). To confirm and compare this result, we numerically integrate the system

$$
\begin{aligned}
& \dot{m}_{i}=-\mu m_{i}+\frac{1}{N} \sum_{j=1}^{N} e^{-|i-j| / N}\left(1-p_{j}(t-T)\right), \\
& \dot{p}_{i}=m_{i}-\mu p_{i},
\end{aligned}
$$

for $N=22, \mu=0.2$, and $T=0$ using MATLAB's built-in function $d d e 23 . m$. Figure 2 shows a summary of the comparison between (29) and the 44-dimensional system (30), where we can see that good agreement was found between both systems as $N$ becomes large. In addition, Figure 2 also presents a time course simulation for $N=22$, 
TABLE 1: Numerical results for $\mu=0.2$ and various $N$.

\begin{tabular}{lccr}
\hline$N$ & $c$ & $\omega$ & $T_{\text {cr }}$ \\
\hline 2 & 1.6065 & 0.87365 & 0.51518 \\
5 & 3.7453 & 0.84206 & 0.55386 \\
10 & 7.4137 & 0.83748 & 0.55982 \\
15 & 11.0992 & 0.83663 & 0.56094 \\
$\mathbf{2 2}$ & $\mathbf{1 6 . 2 6 5 5}$ & $\mathbf{0 . 8 3 6 2 7}$ & $\mathbf{0 . 5 6 1 4 2}$ \\
30 & 22.1729 & 0.83612 & 0.56161 \\
50 & 36.9457 & 0.83601 & 0.56175 \\
100 & 73.8836 & 0.83596 & 0.56181 \\
200 & 147.7634 & 0.83595 & 0.56183 \\
1000 & 738.8111 & 0.83595 & 0.56184 \\
\hline
\end{tabular}
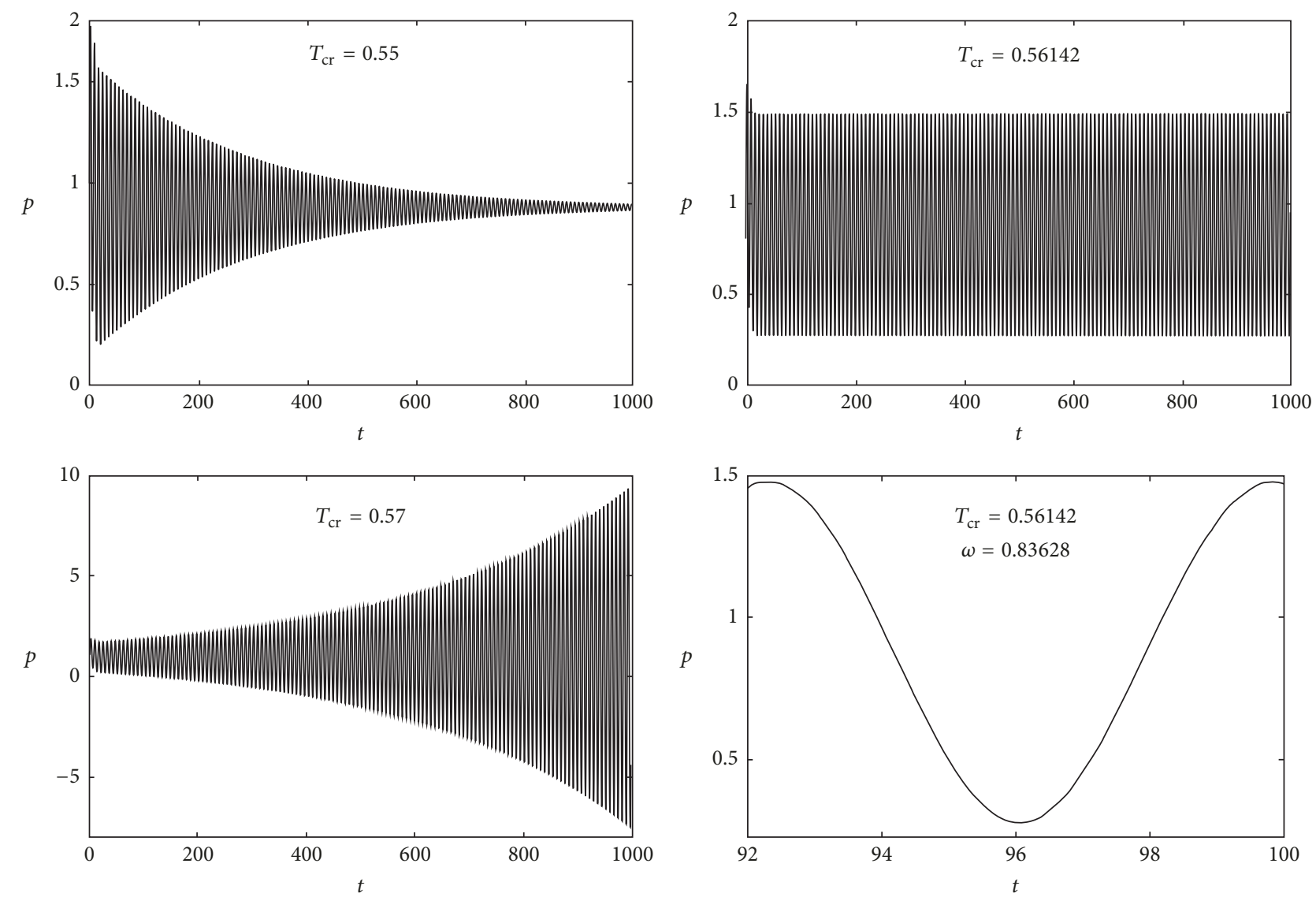

Figure 3: Numerical simulations for $\mu=0.2, N=22$, and different delay values. Here we plotted $p_{1}$, which serves as a representative for the other $21 p_{i}$ 's, since they all exhibit the same time course simulations. We can see that the equilibrium solution is stable for $T=0.55<T_{\mathrm{cr}}$ and unstable for $T=0.57>T_{\mathrm{cr}}$. For $T_{\mathrm{cr}}=0.56142$ the system exhibits oscillations with a frequency $\omega=2 \pi /$ period $=2 \pi /(99.794-92.281)=$ 0.83628 . These are the simulations associated with the $N=22$ case in Table 1.

where we exhibit the short transients to equilibrium for the 22 variables $p_{i}$ for $i=1,2, \ldots, 22$.

Now we use (21) and (22) to compute the critical delay and frequency where the steady state $p^{*}(x)$ loses its stability. For the IDDE system, setting $\mu=0.2$ in (29) gives the values $\omega=0.83595$ and $T_{\text {cr }}=0.56184$, which we show as the limiting value for the DDE system when $N$ becomes large. Table 1 shows the results for $\mu=0.2$ for various values of $N$ and Figure 3 presents the numerical simulations for $\mu=0.2$,
$N=22$, and various delay values using MATLAB's dde23.m. For the case $N=22$, Figure 3 shows that the equilibrium solution is stable for $T=0.55<T_{\text {cr }}$ and unstable for $T=0.57>T_{\text {cr }}$. For $T_{\text {cr }}=0.56142$, the system exhibits oscillations with a frequency $\omega=2 \pi /$ period $=2 \pi /(99.794-$ $92.281)=0.83628$ as predicted. Notice that in Figure 3 we only plotted $p_{1}$, which we use as one of the representatives for the other $21 p_{i}$ 's, since they all exhibit the same time course simulation. Table 1 also shows the approach to the limiting 
value $T_{\text {cr }}=0.56184$ which was approximately achieved in a system of 2000 equations.

\section{Conclusions}

In this work, we investigated the equilibrium solutions and their associated stability of a biological model with negative feedback and time delay. The model is formed of an IDDE coupled to a PDE having time, $t$, and distance, $x$, as independent variables. The study considers linear production and degradation rates of mRNA and protein and an exponential weighting function that models the net repression of all proteins due to spatial distribution in the cytoplasm. Our steady state analysis was accomplished by transforming the steady state integral equation into a second-order two-point boundary value problem, and it showed that the equilibrium solution, $p^{*}$, depends on the distance, $x$. Stability analysis then revealed that the nondelayed system is stable and that there exists a critical value for the delay where the equilibrium loses its stability.

We confirmed our results by "discretizing" our original model and approximating it with a system of $N$ DDEs coupled to $N$ ODEs. This resulted in a $2 N$-dimensional system with delay where numerical evaluations for different $N$ were performed and good agreement was found with the "continuous" IDDE counterpart as $N$ became large. In particular, Table 1 shows that $T_{\mathrm{cr}}^{\text {discrete }} \rightarrow T_{\mathrm{cr}}^{\text {continuous }}=0.56184$ as $N \rightarrow \infty$, which was confirmed using MATLAB's builtin function dde23. $m$ on the full DDE model in a system of 2000 equations. Unfortunately, there are no numerical routines available in MATLAB for the simulation of IDDEs, but our results confirm that it is possible to dissect and understand the dynamics of such complicated equations via a discretization approach, as the one presented in Section 3. The current work corrects and extends our previous study [15] via MATLAB's dde23. $m$ and thus providing more accurate and reliable approximations (and numerical simulations) for the associated $2 \mathrm{~N}$-dimensional system used to confirm our results. Table 1 summarizes and corrects our previous results [15] by showcasing the $N=22$ numerical simulations and their transition from stable to unstable behavior as seen in Figure 3. It is thus hoped that our approach will be useful to researchers in the field of computational mathematics and gene networks trying to model physical or biological systems characterized by IDDEs and PDEs. Future possible directions for this work include choosing $H(p)$ nonlinear, multiple delays, or a detailed bifurcation study proving that the system undergoes a Hopf bifurcation when $T=T_{\mathrm{cr}}$.

\section{Conflicts of Interest}

The author declares that there are no conflicts of interest regarding the publication of this paper.

\section{References}

[1] I. Palmeirim, D. Henrique, D. Ish-Horowicz, and O. Pourquié, "Avian hairy gene expression identifies a molecular clock linked to vertebrate segmentation and somitogenesis," Cell, vol. 91, no. 5, pp. 639-648, 1997.
[2] M. B. Elowitz and S. Leibier, "A synthetic oscillatory network of transcriptional regulators," Nature, vol. 403, no. 6767, pp. 335$338,2000$.

[3] D. Bratsun, D. Volfson, L. S. Tsimring, and J. Hasty, "Delayinduced stochastic oscillations in gene regulation," Proceedings of the National Acadamy of Sciences of the United States of America, vol. 102, no. 41, pp. 14593-14598, 2005.

[4] J. Lewis, "Autoinhibition with transcriptional delay: a simple mechanism for the zebrafish somitogenesis oscillator," Current Biology, vol. 13, no. 16, pp. 1398-1408, 2003.

[5] N. A. M. Monk, "Oscillatory expression of Hes1, p53, and NF$\kappa \mathrm{B}$ driven by transcriptional time delays," Current Biology, vol. 13, no. 16, pp. 1409-1413, 2003.

[6] A. Verdugo and R. Rand, "Hopf bifurcation in a DDE model of gene expression," Communications in Nonlinear Science and Numerical Simulation, vol. 13, no. 2, pp. 235-242, 2008.

[7] H. de Jong, "Modeling and simulation of genetic regulatory systems: a literature review," Journal of Computational Biology, vol. 9, no. 1, pp. 67-103, 2002.

[8] T. Schlitt and A. Brazma, "Current approaches to gene regulatory network modelling," BMC Bioinformatics, vol. 8, no. 6, article no. S9, 2007.

[9] H. De Jong, J.-L. Gouzé, C. Hernandez, M. Page, T. Sari, and J. Geiselmann, "Qualitative simulation of genetic regulatory networks using piecewise-linear models," The Bulletin of Mathematical Biology, vol. 66, no. 2, pp. 301-340, 2004.

[10] A. Mochizuki, "Structure of regulatory networks and diversity of gene expression patterns," Journal of Theoretical Biology, vol. 250, no. 2, pp. 307-321, 2008.

[11] S. Turner, J. A. Sherratt, K. J. Painter, and N. J. Savill, "From a discrete to a continuous model of biological cell movement," Physical Review E, vol. 69, 2004.

[12] J. Goutsias and S. Kim, "Stochastic transcriptional regulatory systems with time delays: A mean-field approximation," Journal of Computational Biology, vol. 13, no. 5, pp. 1049-1076, 2006.

[13] A. Ribeiro, R. Zhu, and S. A. Kauffman, "A general modeling strategy for gene regulatory networks with stochastic dynamics," Journal of Computational Biology, vol. 13, no. 9, pp. 1630$1639,2006$.

[14] R. Edwards, P. Van Den Driessche, and L. Wang, "Periodicity in piecewise-linear switching networks with delay," Journal of Mathematical Biology, vol. 55, no. 2, pp. 271-298, 2007.

[15] A. Verdugo and R. H. Rand, "DDE Model of Gene Expression: A Continuum Approach," in ASME Proceedings of IMECE, pp. 1112-1120, 2008. 


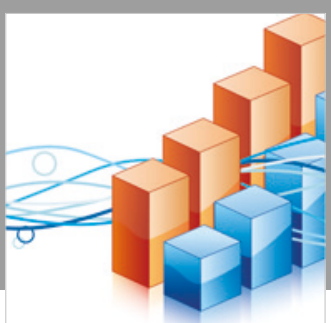

Advances in

Operations Research

\section{-n-m}
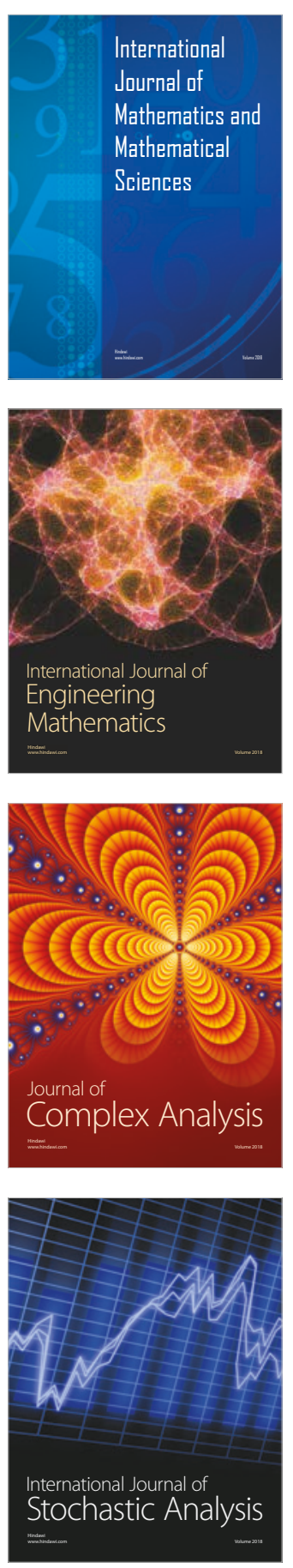
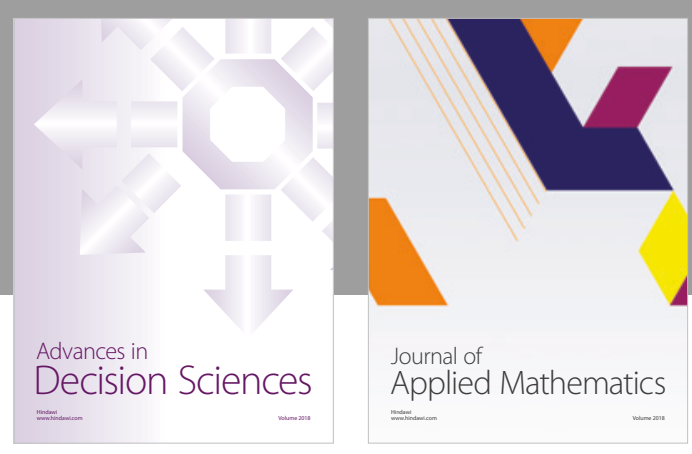

Journal of

Applied Mathematics
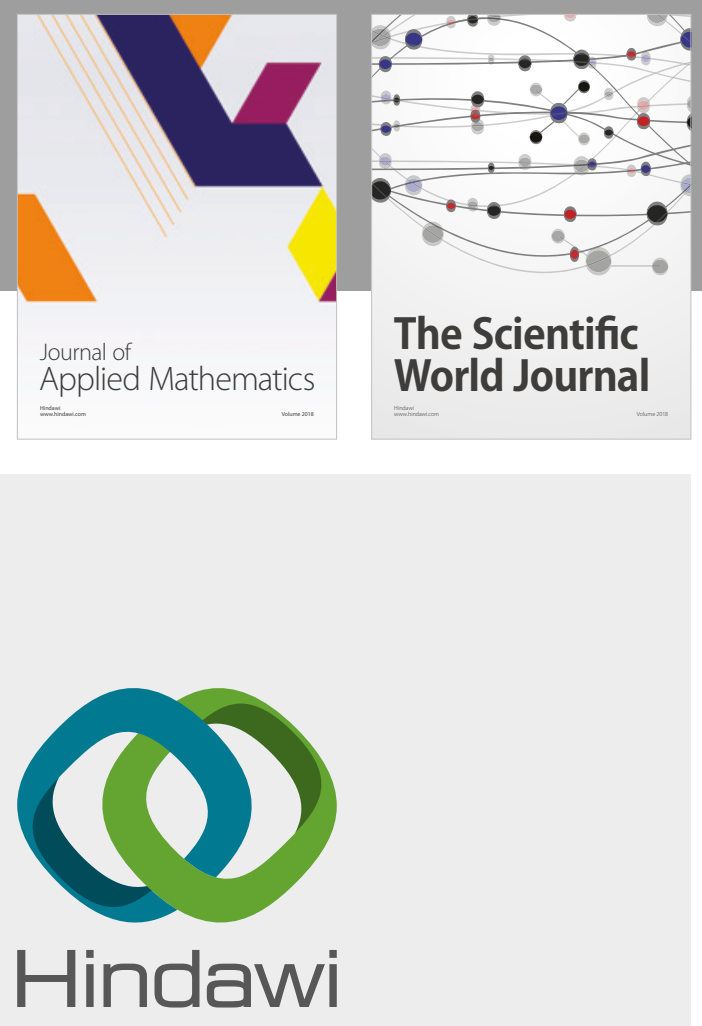

Submit your manuscripts at

www.hindawi.com

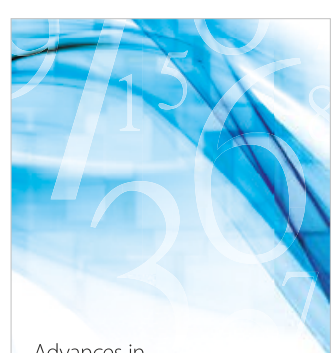

Advances in
Numerical Analysis
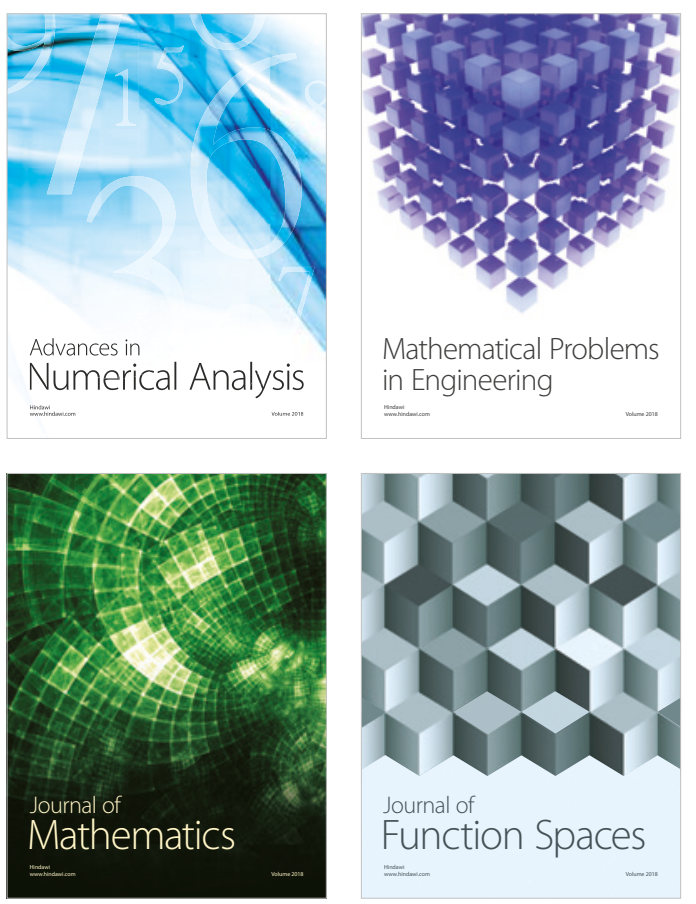

Mathematical Problems in Engineering

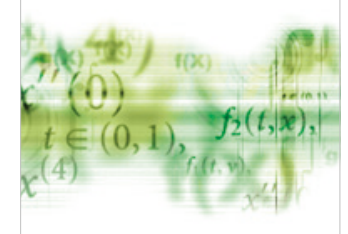

International Journal of

Differential Equations

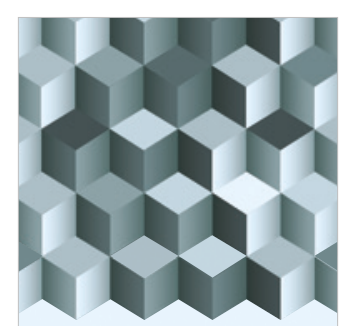

Journal of

Function Spaces

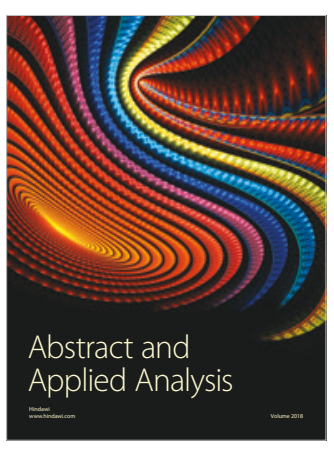

The Scientific

World Journal

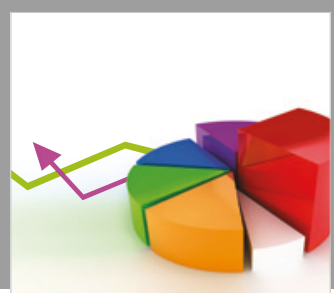

Journal of

Probability and Statistics
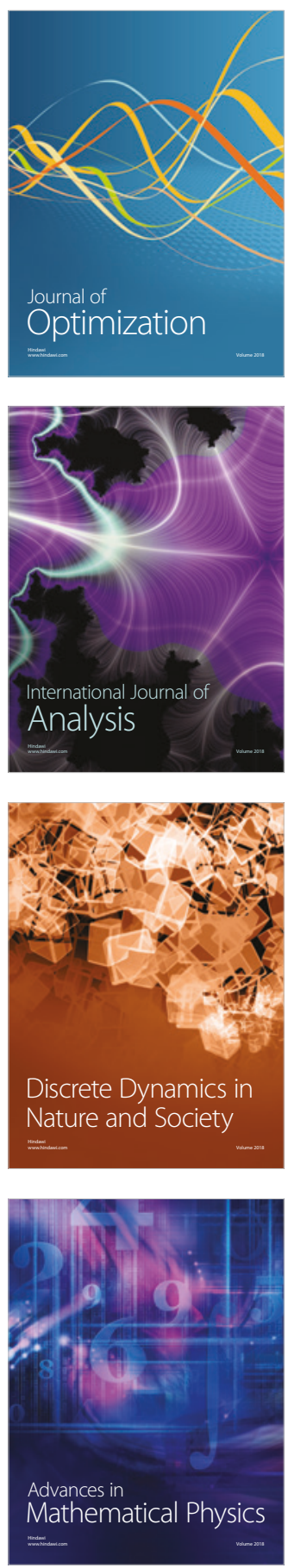